\title{
Spin alignment measurements of vector mesons with ALICE at LHC
}

\section{Bedangadas Mohanty (for the ALICE Collaboration) ${ }^{a, *}$}

${ }^{a}$ National Institute of Science Education and Research, HBNI, Jatni 752050, India

E-mail: bedanga@niser.ac.in

We present the measurements related to the spin alignment of $\mathrm{K}^{* 0}$ and $\phi$ vector mesons at midrapidity for $\mathrm{Pb}-\mathrm{Pb}$ collisions at $\sqrt{s_{\mathrm{NN}}}=2.76 \mathrm{TeV}$ using the ALICE detector at the LHC. The second diagonal spin density matrix element $\left(\rho_{00}\right)$ is measured from the angular distribution of the decay daughters of the vector meson in the decay rest frame, with respect to the event plane and the production plane. The $\rho_{00}$ values are found to be less than $1 / 3$ at low $p_{\mathrm{T}}(<2 \mathrm{GeV} / c)$ for both vector mesons. The observed deviations from $1 / 3$ are maximal for mid-central collisions at a level of $3 \sigma$ for $\mathrm{K}^{* 0}$ and $2 \sigma$ for $\phi$ mesons. As a control measurement the $\rho_{00}$ values were found to be consistent with $1 / 3$, i.e., no significant spin alignment, for pp collisions with respect to the production plane. The results are qualitatively consistent with the expectation from a model that attributes the spin alignment to a polarization of quarks in the presence of large initial angular momentum and a subsequent hadronization by the process of recombination.

40th International Conference on High Energy physics - ICHEP2020

July 28 - August 6, 2020

Prague, Czech Republic (virtual meeting)

${ }^{*}$ Speaker 


\section{Introduction}

The initial stages of high energy heavy-ion collisions at non-zero impact parameter have been theoretically shown to have a large magnetic field [1] and angular momentum [2]. Specifically, the effect of angular momentum, a conserved quantity, is expected to be felt throughout the evolution of the system formed in the collision. One of the physics interests of the heavy-ion program using the ALICE detector at the LHC is to look for signatures of the spin-orbit interactions. This can be studied by measuring the angular distributions of the decay daughters of hyperons and vector mesons relative to the system's angular momentum direction [3-5].

The angular distributions are measured with respect to a quantization axis, which can either be perpendicular to the production plane of the vector meson, or normal to the reaction plane of the system. The normal direction is expected to be the direction of the angular momentum for the system. The production plane is defined by the momentum of the vector meson under study and the beam direction, whereas the reaction plane is defined by the impact parameter and beam direction. The spin alignment of a vector meson is described by a $3 \times 3$ Hermitian spin-density matrix [8]. The matrix elements can be obtained by measuring the angular distributions of the decay products of the vector mesons with respect to a quantization axis. The angle denoted as $\theta^{*}$ is that defined by one of the decay daughters of the vector meson in the rest frame of the vector meson with respect to the quantization axis. In general, the angular distribution for vector mesons is expressed as [7]

$$
\frac{\mathrm{d} N}{\mathrm{~d} \cos \theta^{*}}=N_{0}\left[1-\rho_{00}+\cos ^{2} \theta^{*}\left(3 \rho_{00}-1\right)\right],
$$

$N_{0}$ is a normalization constant, $\rho_{00}$ is the second diagonal element of the spin density matrix. The spin 1 vector mesons can be in three spin states of $-1,0$ and 1 . The $\rho_{00}$ defines the probability of finding a vector meson in spin state zero. The $\rho_{00}$ is $1 / 3$ in the absence of spin alignment and the angular distribution in Eq. 1 is uniform. If initial conditions or the final hadronization process cause polarization effects in heavy-ion collisions, then the angular distributions as defined in Eq. 1 would become non uniform. This would lead to $\rho_{00}$ values being different from $1 / 3$. This is the experimental signature of spin alignment.

In this contribution, we present the results at LHC energies related to the spin alignment of $\mathrm{K}^{* 0}$ and $\phi$ vector mesons through the measurement of $\rho_{00}$ in pp and $\mathrm{Pb}-\mathrm{Pb}$ collisions with respect to the production plane (PP) and event plane (EP, an experimental measure of the reaction plane).

\section{Analysis details}

The analyses are carried out using 14 million minimum bias $\mathrm{Pb}-\mathrm{Pb}$ collisions at $\sqrt{s_{\mathrm{NN}}}=2.76$ $\mathrm{TeV}$, collected in the year 2010 and 43 million minimum bias pp collisions at $\sqrt{s}=13 \mathrm{TeV}$, taken in the year 2015. The measurements for vector mesons are performed at midrapidity $(|y|<0.5)$ as a function of the transverse momentum $p_{\mathrm{T}}$ and for different centrality classes in $\mathrm{Pb}-\mathrm{Pb}$ collisions. The $\mathrm{K}_{\mathrm{S}}^{0}$ analysis is performed only for $\mathrm{Pb}-\mathrm{Pb}$ collisions in the $20-40 \%$ centrality class as a null hypothesis test, as it has spin zero. The details of the ALICE detector, trigger conditions, centrality selection, and second order event plane estimation using the V0 detectors at forward rapidity, can be found in [9-12]. 
The $\mathrm{K}^{* 0}$ and $\phi$ vector mesons are reconstructed via their decays into charged $\mathrm{K} \pi$ and $\mathrm{KK}$ pairs, respectively, while the $\mathrm{K}_{\mathrm{S}}^{0}$ is reconstructed via its decay into two pions. The Time Projection Chamber (TPC) [13] and Time-of-Flight (TOF) detector [14] are used to identify the decay products of these mesons via specific ionization energy loss and time-of-flight measurements, respectively. The meson yields are determined via the invariant mass technique [15-18]. The background coming from combinatorial pairs and misidentified particles is removed by constructing the invariant mass distribution from the mixed events [15-18].

The invariant mass distributions are fitted with a Breit-Wigner (Voigtian: convolution of BreitWigner and Gaussian distributions) function for the $\mathrm{K}^{* 0}(\phi)$ signal and a second order polynomial that describes the residual background, in order to extract the yields [15-17]. Extracted yields are then corrected for the reconstruction efficiency and acceptance in each $\cos \theta^{*}$ and $p_{\mathrm{T}}$ bin [1517]. The resulting efficiency and acceptance corrected $\mathrm{d} N / \mathrm{d} \cos \theta^{*}$ distributions are fitted with the functional form given in Eq. 1 to determine $\rho_{00}$ for each $p_{\mathrm{T}}$ bin in $\mathrm{pp}$ and $\mathrm{Pb}-\mathrm{Pb}$ collisions [19]. For the EP results, the finite resolution of the event plane is corrected [9].

There are three main sources of systematic uncertainties in the measurements of the angular distribution of vector meson decays, which are propagated to $\rho_{00}$ : (a) Meson yield extraction procedure. These sources contribute to the uncertainties on the $\rho_{00}$ value at a level of 12(8)\% at the lowest $p_{\mathrm{T}}$ and decrease with $p_{\mathrm{T}}$ to $4(3) \%$ at the highest $p_{\mathrm{T}}$ studied for the $\mathrm{K}^{* 0}(\phi)$. (b) Track selection criteria. The systematic uncertainties on the $\rho_{00}$ value due to variation on the track selection criteria are $14(6) \%$ at the lowest $p_{\mathrm{T}}$ and about $11(5) \%$ at the highest $p_{\mathrm{T}}$ for $\mathrm{K}^{* 0}(\phi)$. (c) Particle identification procedure. The corresponding uncertainty is $5(3) \%$ at the lowest $p_{\mathrm{T}}$ and about $4(4.5) \%$ at the highest $p_{\mathrm{T}}$ studied for $\mathrm{K}^{* 0}(\phi)$. The total systematic uncertainty on $\rho_{00}$ is obtained by adding all the contributions in quadrature and details can be found in Ref. [19].

\section{Results and discussions}

Figure 1 shows the measured $\rho_{00}$ as a function of $p_{\mathrm{T}}$ for $\mathrm{K}^{* 0}$ and $\phi$ mesons in pp and $\mathrm{Pb}-\mathrm{Pb}$ collisions, along with the measurements for $\mathrm{K}_{\mathrm{S}}^{0}$ in $\mathrm{Pb}-\mathrm{Pb}$ collisions. In mid-central (10-50\%) Pb- $\mathrm{Pb}$ collisions, $\rho_{00}$ is below $1 / 3$ at the lowest measured $p_{\mathrm{T}}$ and increases to $1 / 3$ within uncertainties for $p_{\mathrm{T}}>2 \mathrm{GeV} / c$. At low $p_{\mathrm{T}}$, the central value of $\rho_{00}$ is smaller for $\mathrm{K}^{* 0}$ than for $\phi$, although the results are compatible within uncertainties. In pp collisions, $\rho_{00}$ is independent of $p_{\mathrm{T}}$ and equal to $1 / 3$ within uncertainties. For the spin zero hadron $\mathrm{K}_{\mathrm{S}}^{0}, \rho_{00}$ is consistent with $1 / 3$ within uncertainties in $\mathrm{Pb}-\mathrm{Pb}$ collisions. The results with random event plane directions are also compatible with no spin alignment for the studied $p_{\mathrm{T}}$ range. The results for the random production plane (the momentum vector direction of each vector meson is randomized) are similar to random event plane measurements.

Figure 2 shows $\rho_{00}$ for $\mathrm{K}^{* 0}$ and $\phi$ mesons as a function of average number of participating nucleons $\left(\left\langle N_{\text {part }}\right\rangle\right)$ [12] for Pb-Pb collisions at $\sqrt{s_{\mathrm{NN}}}=2.76 \mathrm{TeV}$. Large $\left\langle N_{\text {part }}\right\rangle$ correspond to the central collisions, while peripheral events have low $\left\langle N_{\text {part }}\right\rangle$. In the lowest $p_{\mathrm{T}}$ range, the $\rho_{00}$ values have maximum deviation from $1 / 3$ for intermediate centrality (20-40\%) and approach $1 / 3$ for both central and peripheral collisions. At higher $p_{\mathrm{T}}$, the $\rho_{00}$ measurements are consistent with $1 / 3$ for all the collision centrality classes studied for both vector mesons. For the low- $p_{\mathrm{T}}$ measurements in mid-central $\mathrm{Pb}-\mathrm{Pb}$ collisions, the maximum deviations of $\rho_{00}$ from $1 / 3$ are 3.2(2.6) $\sigma$ and 2.1(1.9) $\sigma$ 


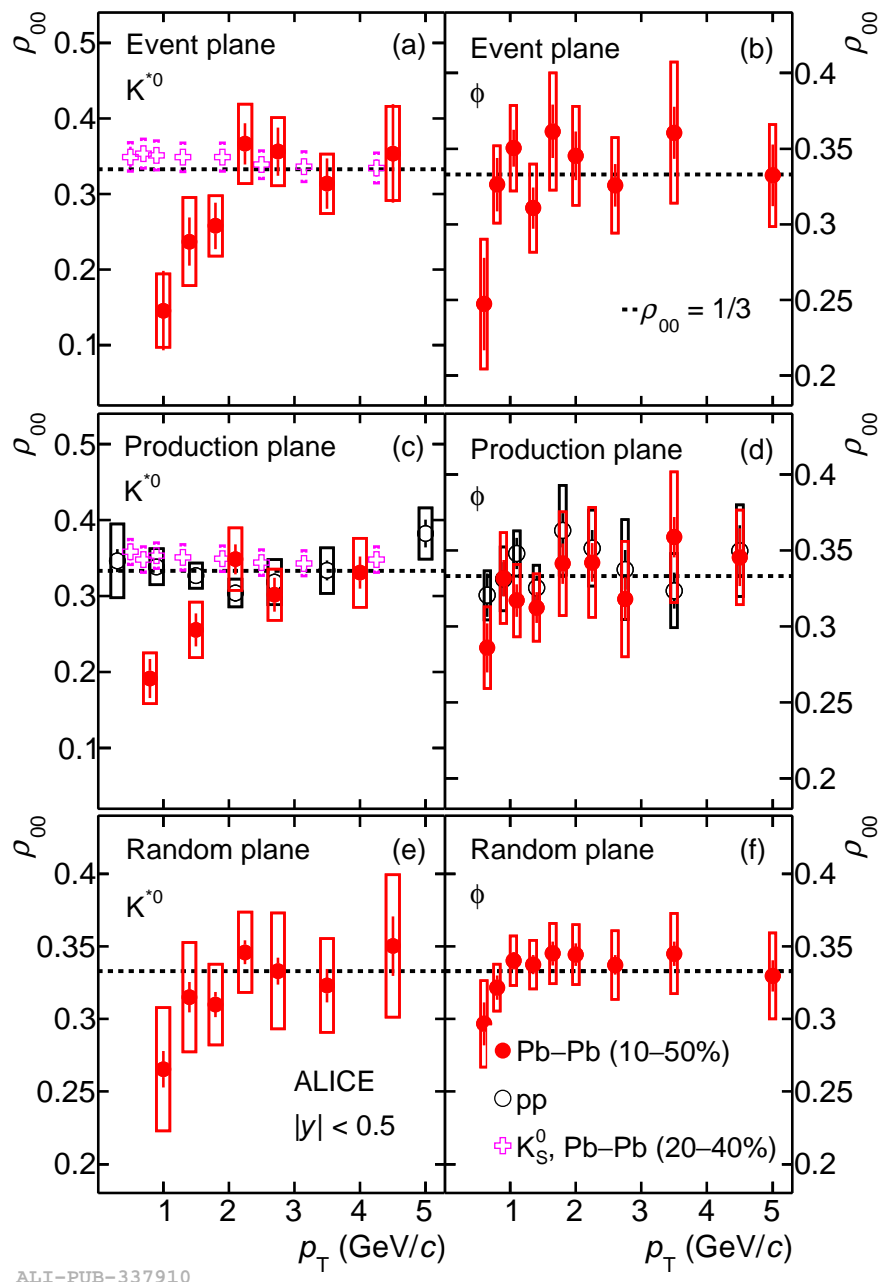

Figure 1: The measured $\rho_{00}$ as a function of $p_{\mathrm{T}}$ corresponding to $\mathrm{K}^{* 0}, \phi$, and $\mathrm{K}_{\mathrm{S}}^{0}$ mesons at $|y|<0.5$ in $\mathrm{Pb}-\mathrm{Pb}$ collisions at $\sqrt{s_{\mathrm{NN}}}=2.76 \mathrm{TeV}$ and minimum bias pp collisions at $\sqrt{s}=13 \mathrm{TeV}$. Results are shown for spin alignment with respect to event plane (panels a,b), production plane (c,d) and random event plane (e,f) for $\mathrm{K}^{* 0}$ (left column) and $\phi$ (right column) [19]. The statistical and systematic uncertainties are shown as bars and boxes, respectively.

for $\mathrm{K}^{* 0}$ and $\phi$ mesons, respectively, for mid-central (20-40\%) $\mathrm{Pb}-\mathrm{Pb}$ collisions with respect to the PP (EP). The $\sigma$ are calculated by adding statistical and systematic uncertainties in quadrature.

The qualitative theory predictions for the spin alignment effect are the following [20]: (i) $\rho_{00}>1 / 3$ if the hadronization of a polarized parton proceeds via fragmentation, (ii) $\rho_{00}<1 / 3$ for hadronization of polarized parton via recombination, (iii) $\rho_{00}$ is expected to have a maximum deviation from $1 / 3$ for mid-central heavy-ion collisions, which is consistent with angular momentum being maximal, and a smaller deviation for both peripheral (large impact parameter and small $\left.\left\langle N_{\text {part }}\right\rangle\right)$ and central (small impact parameter and large $\left\langle N_{\text {part }}\right\rangle$ ) collisions, (iv) the $\rho_{00}$ value is expected to have a largest deviation from $1 / 3$ at low $p_{\mathrm{T}}$ and reach the value of $1 / 3$ at high $p_{\mathrm{T}}$ in the recombination hadronization scenario, and (v) the effect is expected to be larger for $\mathrm{K}^{* 0}$ compared to $\phi$ due to their constituent quark composition. All of these features are probed experimentally for $\mathrm{K}^{* 0}$ and 


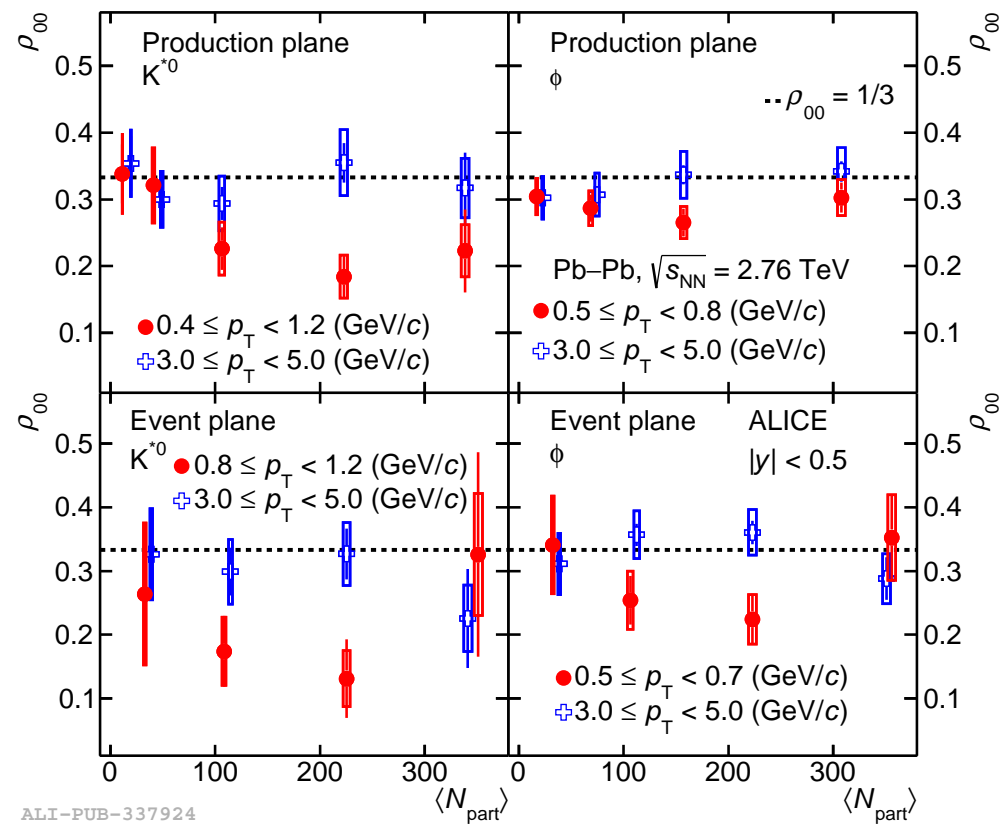

Figure 2: The measured $\rho_{00}$ as a function of $\left\langle N_{\text {part }}\right\rangle$ for $\mathrm{K}^{* 0}$ and $\phi$ mesons at low and high $p_{\mathrm{T}}$ in $\mathrm{Pb}-\mathrm{Pb}$ collisions [19]. The statistical and systematic uncertainties are shown as bars and boxes, respectively.

$\phi$ vector mesons in $\mathrm{Pb}-\mathrm{Pb}$ collisions [19] and reported in this contribution. The experimental results indicate that a spin alignment is present at lower $p_{\mathrm{T}}$, which is qualitatively consistent with the predictions [20]. This centrality dependence is qualitatively consistent with the dependence of initial angular momentum on impact parameter in heavy-ion collisions [2].

Significant polarization of $\Lambda$ baryons (spin $=1 / 2$ ) was reported at low RHIC energies. The polarization is found to decrease with increasing $\sqrt{s_{\mathrm{NN}}}$ [4]. At the LHC energies, the global polarization for $\Lambda$ baryons was measured to be compatible with zero within uncertainties [6]. In the recombination model, $\rho_{00}$ is expected to depend on the square of the quark polarization whereas the $\Lambda$ polarization depends linearly on it, therefore using quark polarization information from $\Lambda$ measurements will yield a $\rho_{00} \sim 1 / 3$ at LHC energies. The large effect observed for $\mathrm{K}^{* 0}$ and $\phi$ vector mesons for the central value of $\rho_{00}$ for mid-central $\mathrm{Pb}-\mathrm{Pb}$ collisions at low $p_{\mathrm{T}}$ is therefore puzzling. However, the magnitude of the spin alignment also depends on the details of the transfer of the quark polarization to the hadrons (baryon vs. meson), details of the hadronization mechanism (recombination vs. fragmentation), re-scattering, regeneration, and possibly the lifetime and mass of the hadrons in the system. Moreover, the vector mesons are predominantly primordially produced whereas the hyperons are expected to have large contributions from resonance decays.

\section{Summary}

We observe a significant spin alignment effect for $\mathrm{K}^{* 0}$ and $\phi$ mesons in heavy-ion collisions. The measured spin alignment of the vector mesons is surprisingly large compared to the polarization measured for $\Lambda$ hyperons. The effect is strongest when the alignment is measured at low $p_{\mathrm{T}}$ with 
respect to a vector perpendicular to the reaction plane and for mid-central (10-50\%) collisions. These observations are qualitatively consistent with expectations from the effect of large initial angular momentum in non-central heavy-ion collisions, which leads to quark polarization via spinorbit coupling and is subsequently transferred to hadronic degrees of freedom by hadronization via recombination. Results with increased statistical precision are expected for spin alignment studies with the $\mathrm{Pb}-\mathrm{Pb}$ data set at $\sqrt{s_{\mathrm{NN}}}=5.02 \mathrm{TeV}$. This will help in arriving at a better significance of the observed effects. In future measurements, the difference in the polarization of $\mathrm{K}^{* \pm}$ and $\mathrm{K}^{* 0}$, due to their different magnetic moments, would be directly sensitive to the effect of the large initial magnetic field produced in heavy-ion collisions.

\section{References}

[1] D. E. Kharzeev, L. D. McLerran and H. J. Warringa, Nucl. Phys. A 803, 227 (2008).

[2] F. Becattini, F. Piccinini and J. Rizzo, Phys. Rev. C 77, 024906 (2008).

[3] B. I. Abelev et al. [STAR Collaboration], Phys. Rev. C 76, 024915 (2007).

[4] L. Adamczyk et al. [STAR Collaboration], Nature 548, 62 (2017).

[5] B. I. Abelev et al. [STAR Collaboration], Phys. Rev. C 77, 061902 (2008).

[6] S. Acharya et al. [ALICE], Phys. Rev. C 101, no.4, 044611 (2020).

[7] K. Schilling, P. Seyboth and G. E. Wolf, Nucl. Phys. B 15, 397 (1970).

[8] Y. G. Yang, R. H. Fang, Q. Wang and X. N. Wang, Phys. Rev. C 97, no.3, 034917 (2018).

[9] B. B. Abelev et al. [ALICE], JHEP 06, 190 (2015).

[10] K. Aamodt et al. [ALICE], JINST 3, S08002 (2008).

[11] K. Aamodt et al. [ALICE], Phys. Rev. Lett. 106, 032301 (2011).

[12] B. Abelev et al. [ALICE], Phys. Rev. C 88, no.4, 044909 (2013).

[13] J. Alme et al. Nucl. Instrum. Meth. A 622, 316-367 (2010).

[14] G. Dellacasa et al. [ALICE], CERN-LHCC-2000-012.

[15] J. Adam et al. [ALICE], Phys. Rev. C 95, no.6, 064606 (2017).

[16] B. B. Abelev et al. [ALICE], Phys. Rev. C 91, 024609 (2015).

[17] S. Acharya et al. [ALICE], Phys. Lett. B 802, 135225 (2020).

[18] B. B. Abelev et al. [ALICE], Phys. Rev. Lett. 111, 222301 (2013).

[19] S. Acharya et al. [ALICE], Phys. Rev. Lett. 125, no.1, 012301 (2020).

[20] Z. T. Liang and X. N. Wang, Phys. Lett. B 629, 20-26 (2005). 